\title{
The Synapse Project: Engagement in mentally challenging activities enhances neural efficiency
}

\author{
Ian M. McDonough, Sara Haber, Gérard N. Bischof and Denise C. Park* \\ Center for Vital Longevity, School of Behavioral and Brain Sciences, University of Texas at Dallas, \\ Dallas, TX, USA
}

\begin{abstract}
.
Purpose: Correlational and limited experimental evidence suggests that an engaged lifestyle is associated with the maintenance of cognitive vitality in old age. However, the mechanisms underlying these engagement effects are poorly understood. We hypothesized that mental effort underlies engagement effects and used fMRI to examine the impact of high-challenge activities (digital photography and quilting) compared with low-challenge activities (socializing or performing low-challenge cognitive tasks) on neural function at pretest, posttest, and one year after the engagement program.

Methods: In the scanner, participants performed a semantic-classification task with two levels of difficulty to assess the modulation of brain activity in response to task demands.

Results: The High-Challenge group, but not the Low-Challenge group, showed increased modulation of brain activity in medial frontal, lateral temporal, and parietal cortex - regions associated with attention and semantic processing - some of which were maintained a year later. This increased modulation stemmed from decreases in brain activity during the easy condition for the High-Challenge group and was associated with time committed to the program, age, and cognition.

Conclusions: Sustained engagement in cognitively demanding activities facilitated cognition by increasing neural efficiency. Mentally-challenging activities may be neuroprotective and an important element to maintaining a healthy brain into late adulthood.
\end{abstract}

Keywords: Cognitive engagement, fMRI, intervention, learning, semantic processing

\section{Introduction}

Correlational evidence increasingly suggests that maintaining cognitive health in older adults is associated with engagement in cognitively-challenging activities in middle and old age (Mueller et al., 2013; Singh-Manoux et al., 2003; Wilson et al., 2003). Moreover, correlational analyses have linked greater self-reported cognitive engagement in every-

\footnotetext{
${ }^{*}$ Corresponding author: Denise C. Park, Ph.D., Center for Vital Longevity, 1600 Viceroy Avenue, University of Texas at Dallas, Dallas, TX 75235, USA. Tel.: +1 972883 3255; Fax: +1 972883 3250; E-mail: denise@utdallas.edu.
}

day life to a decreased risk of being diagnosed with Alzheimer's disease (Wilson et al., 2002, 2007) and to a decreased accumulation of beta-amyloid-a precursor of Alzheimer's disease (Landau et al., 2012; Wirth et al., 2014a, b). Together, these results are consistent with the popular adage, "Use it or lose it," that emphasizes how cognitive engagement can prevent cognitive decline and even dementia (e.g., Hertzog et al., 2009; Hultsch et al., 1999; Salthouse, 2006).

Few experimental studies, however, have tested the impact of real-world engagement on cognition and such studies are essential to establish a causal relationship between engagement and cognition (see Stine-Morrow \& Basak, 2011). One of few such 
studies is the Experience Corps program in which older adults spent sustained periods of time partnering with elementary school students, teaching them literacy skills, library support, and classroom etiquette (Carlson et al., 2008, 2009). When compared to a waitlist control group, the older adults who participated in the program showed improvement in episodic memory and executive function. More recently, the Synapse Project investigated the hypothesis that sustained engagement in activities that required new learning and cognitive challenge would facilitate cognition due to expenditure of mental effort and self-initiated processing (Park et al., 2014). Results indicated that older adults who engaged in cognitively-demanding novel activities (e.g., learning digital photography or quilting) for 15 hours a week over a period of 3 months showed improved episodic memory and some evidence of improved speed of processing compared with control groups that participated in activities requiring little new learning, such as socializing or listening to classical music. In the present paper, we present functional neuroimaging data from pretest to posttest in a subset of participants who participated in the Synapse Project,. We also collected one year follow-up, data to determine the durability of any observed effects due to engagement.

How might sustained cognitive engagement support neural function? Substantial research, primarily in animals, indicates that neurogenesis and manifestation of synaptic plasticity are more likely to occur under conditions of high cognitive demands (for recent reviews, see Buitenweg et al., 2012; Park \& Bischof, 2013; Shors et al., 2012). A recent review by Shors (2014) emphasized that animals engaged in demanding, novel learning showed long-term survival of fragile neurons resulting from neurogenesis. Shors suggested that longer learning periods would confer greater protection of these new neurons, which would ultimately enhance cognition. The Synapse Project was an effort to translate these ideas from animal research to humans by affecting changes in lifestyle engagement that varied demands on mental effort.

The benefits of demanding, novel learning on the brain might be particularly pronounced for older adults, who exhibit clear declines in brain regions supporting memory and other cognitive processes (for reviews, see Grady, 2012; Greenwood, 2007; Park \& Reuter-Lorenz, 2009). For example, during memory encoding, older adults under-recruit brain activity in the prefrontal cortex and lateral temporal regions com- pared to younger adults (Kirchhoff et al., 2012; Logan et al., 2002). These under-recruited brain regions are thought to reflect deficits in semantic elaboration strategies that are critical for memory performance. More generally, older adults appear to have difficulties initiating semantic elaboration strategies, possibly because of a reduced capacity to modulate brain activity in response to task demands (e.g., Reuter-Lorenz \& Cappell, 2008). Older adults have been shown to reach a neural "ceiling" of activation earlier than younger adults in the face of a cognitive challenge. This activation ceiling has been closely tied to age-related deficits in cognitive processing (e.g., Schneider-Garces et al., 2010).

These neuroimaging findings support decades of behavioral research showing that older adults have difficulty initiating elaborative semantic strategies to the same extent as younger adults (e.g., Craik \& Byrd, 1982; Craik \& Jennings, 1992; Kirchhoff et al., 2012; Park et al., 1990). Critically, neuroimaging studies also have shown that deficits in brain activity can be improved when older adults were guided to use semantic elaboration strategies (Kirchhoff et al., 2012; Logan et al., 2002), suggesting that deficits in brain functioning in these regions can be affected by experience. It is less clear whether demanding, but broad, cognitive engagement can improve deficits in brain functioning to either enhance semantic elaboration or more generally increase modulation of brain activity in the face of a cognitive challenge.

\subsection{The present study}

We used fMRI to assess whether engagement (via quilting, digital photography, or a combination of both) can enhance the ability to modulate neural response to difficulty. In the fMRI task, older adults made living/non-living judgments towards that were either easy (concrete) or hard (ambiguous). A recently published study (Kennedy et al., 2015) utilizing this task showed that judging harder words was associated in younger adults with an increase in modulation of brain activity in frontal and parietal regions implicated in semantic and controlled processing. Older adults, however, showed less modulation during the task in lateral frontal, parietal, and temporal regions consistent with previous work showing age-related reductions in brain modulation (e.g., Reuter-Lorenz \& Cappell, 2008; Schneider-Garces et al., 2010). Thus, this task allowed us to assess the extent that high-challenge engagement 
would increase modulation in brain activity by way of enhancing semantic elaboration strategies. We hypothesized that after participating in a learning environment in which they engaged in highly challenging activities for 14 weeks, older adults would be guided towards spontaneously initiating the mental effort required for novel learning and that this effort would result in a heightened ability to modulate neural response to task demands (i.e., a more youth-like brain state; Nyberg et al., 2012; Park \& Reuter-Lorenz, 2009).

We tested 39 participants from the original Synapse Project, all of whom underwent a battery of cognitive tests and brain scanning using fMRI before (pretest) and after the intervention (posttest). A smaller sample $(N=26)$ also was tested one year after the intervention (follow-up). We predicted that increased modulation of brain activity from easy to harder levels of task demand would be found in bilateral frontal and parietal brain regions involved in controlled processing as well as lateral temporal regions involved in semantic elaboration, replicating the increases in brain activity from easy to hard conditions observed by Kennedy et al. (2015).

We considered two possible patterns of changes in modulation of brain activity resulting from the cognitive engagement manipulation. One possibility is that high-challenge engagement could result in greater modulation of brain activity to difficulty distributed across lateral frontal, parietal, and temporal regions compared with low-challenge engagement. A second possible pattern was that high-challenge engagement could increase modulation of brain activity in new brain regions. Note that these two patterns are not mutually exclusive and engagement could lead to both increased modulation of brain activity in regions showing age-related decline as well as increased brain activity in new brain regions not typically used by young adults. We also conducted analyses to test the extent that the engagement-related gains in brain activity 1 ) would be positively associated with greater time invested in the program, 2) would be positively correlated with changes in cognition, 3 ) would be related to age, and 4) would be maintained after one year.

\section{Materials and methods}

\subsection{Participants}

We collected fMRI data at pretest and posttest from 39 participants out of the original 221 participants who were included in the behavioral Synapse Project, and also collected data from 26 of the 39 participants one year later. Of the 221 behavioral participants, 110 were fMRI eligible. Of those eligible, 55 agreed to be scanned at pretest. After pretest scanning, 13 participants were excluded for the following reasons: scanner malfunction (1), scanner artifact (6), and claustrophobia or vertigo (6). Of these 42 participants with viable scans, three were excluded before posttest because they were no longer eligible for fMRI due to stroke (1), medication (1), and surgery (1), resulting in a sample of 39 participants whose data are reported here. Of the 39 participants that were scanned initially at pretest and posttest, 26 were scanned again one year later. Thirteen participants were not scanned or included in the analyses after one year for the following reasons: were no longer eligible due to MRI exclusions (4), declined to participate (7), could not be contacted (1), and had severe scanner artifact (1). We compared Synapse individuals who participated in the fMRI portion of the study relative to those who did not participate in this portion across a number of demographic and health variables. People in the fMRI study were 3.38 years younger $(p=0.008)$, had 1.13 more years of education $(p=0.016)$, and had higher physical functioning $(p<0.001)$. These differences are consistent with the fact that participants who were eligible were healthier and better educated than non-eligible subjects.

Inclusion criteria for the Synapse project was as follows: age of 60 to 90 , at least a tenth-grade education, fluent English, worked or performed volunteer activities for no more than 10 hours per week, were novices at digital photography and quilting, and reported no more than 10 hours per week of web surfing and computer activity. Participants also had visual acuity (or corrected acuity) of 20/40, a minimum score of 26 on the Mini-Mental Examination (Folstein et al., 1975), had no known cardiovascular or neurological disorders, and had no history of drug and alcohol abuse. Additional MRI eligibility requirements included being right handed, and no known claustrophobia.

To recruit participants, an information session was held in which all six groups were described. Participants were required to agree to be randomly assigned to five of the groups and were allowed to exclude assignment to the photo, quilt, or dual condition. We included the exclusion to ensure that participants would perform an activity of some interest to them to minimize dropout rate. More detailed recruitment information can be found elsewhere (Park et al., 2014). 
Table 1

Demographic information for participants in all groups

\begin{tabular}{lcccccc}
\hline Group & N (Initial/1Yr) & Age & Sex (F/M) & Yrs Education & MMSE & Adherence Hours \\
\hline High Challenge & $23 / 16$ & $68.74(1.32)$ & $17 / 6$ & $16.98(0.64)$ & $29.04(0.93)$ & $221.88(8.36)$ \\
Photo & $7 / 4$ & $70.23(1.86)$ & $5 / 2$ & $18.43(1.02)$ & $29.29(0.36)$ & $218.25(9.64)$ \\
Quilt & $7 / 6$ & $70.71(2.72)$ & $6 / 1$ & $15.93(0.79)$ & $28.71(0.42)$ & $228.89(10.84)$ \\
Dual & $9 / 6$ & $66.00(2.09)$ & $6 / 3$ & $16.67(1.23)$ & $29.11(0.26)$ & $219.25(19.03)$ \\
Low Challenge & $16 / 10$ & $69.31(1.45)$ & $12 / 4$ & $16.88(0.81)$ & $29.12(1.09)$ & $224.45(8.65)$ \\
Social & $9 / 6$ & $70.22(2.13)$ & $7 / 2$ & $17.56(1.17)$ & $29.22(0.28)$ \\
Placebo & $7 / 4$ & $68.14(1.98)$ & $5 / 2$ & $16.00(1.09)$ & $29.00(0.53)$ & $231.71(19.83)$ \\
\hline
\end{tabular}

Notes. Standard Error of the Mean in parentheses. 1 Yr = One-year follow up, Yrs = Years, MMSE = Mini Mental State Exam.

Participants in this study were assigned to one of two groups: the High-Challenge group $(n=23)$ or the LowChallenge group $(n=16)$. The High-Challenge group included seven participants from the Photo group, seven from the Quilting group, and nine from the Dual group (see below for group descriptions). The LowChallenge group was comprised of nine participants from the Social group and seven from the Placebo group. The larger number of participants in the HighChallenge group is due to the fact that there were three High-Challenge groups of volunteers (photo, quilting, dual) and two Low-Challenge, but active groups (social and placebo). The ratios of volunteers for those who participated in the larger study and those included in the fMRI portion did not significantly differ, $\chi^{2}(1$, $n=257)=0.096, p=0.76$, suggesting that the selection effects were not greater for the Low-Challenge than the High-Challenge group. Demographic information for all participants included in this study can be found in Table 1.

\subsection{Procedures}

\subsubsection{Intervention}

The High-Challenge group spent at least 15 hours per week over a 14-week period on group-related activities. Activities required sustained cognitive challenge, as participants were required to learn progressively more difficult skills in digital photography, quilting, or a combination of both (i.e., the dual group). Participants were instructed by a professional instructor in groups of six or less in classes that met for 2.5 hours, twice a week. Additionally, participants spent at least 10 hours per week at the site working on individual projects related to their group assignment. For those that learned digital photography, engagement activities focused on the acquisition and practical application of computer and photography skills. They started by learning basic camera operations and progressed to using high level editing software and photography techniques. For those that learned quilting, participants initially mastered the basic operations of the computerdriven sewing machines and then progressed from basic to more intricate quilting techniques that utilized design of complex abstract patterns. Participants assigned to the Dual group engaged for seven weeks in digital photography and seven weeks in quilting, with the ordering of the engagement counterbalanced across participants.

The Low-Challenge group spent at least 15 hours per week over a 14-week period on their activities. In contrast to the High-Challenge group, they experienced minimal new learning with no active skill acquisition. The Low-Challenge group was comprised of participants in the Social group, which met for 15 hours per week to socialize and engage in themed activities related to travel, cooking, etc. with no active learning component. The Placebo group engaged in low-demand cognitive tasks such as listening to music, playing simple games, or watching classic movies.

\subsubsection{MRI scanner task}

The semantic judgment task consisted of one functional run that lasted approximately seven minutes. Using a blocked design, participants viewed a series of 128 nouns for $2500 \mathrm{~ms}$ each and judged whether each noun referred to a living or non-living item with a button press (yes or no). A $500 \mathrm{~ms}$ inter-trial interval was filled with a fixation cross between each word. Half the words were living and half non-living. Within each category, half were easy to classify (e.g., LION or RADIO) and half were harder to classify in that they were ambiguous and had some characteristics of both living and non-living things (e.g., VIRUS or ZOMBIE). We note that the purpose of this manipulation was to develop a task that would require more 
neural resources than the easy task (increased modulation) and take longer to complete, effects that were observed by Kennedy et al. (2015) when they used this task. The increase in difficulty was intentionally multidimensional in that increased judgment time was required for multiple reasons including the ambiguity of the word as well as the lower word frequency and decreased concreteness of difficult items relative to easy items. The words were divided into 8 easy and 8 hard blocks of words. Each block contained 8 words and lasted for $24 \mathrm{~s}$. An additional set of 3 blocks assessed baseline brain activity by presenting only a fixation cross $(24 \mathrm{~s})$. Blocks were presented in a pseudorandom order.

\subsubsection{Cognitive battery}

The cognitive battery administered at pretest, posttest, and at one-year follow-up consisted of three to four measures of processing speed, episodic memory, mental control, and visuospatial processing, which were used to form constructs, as described in Park et al. (2014). The cognitive results in the full sample $(N=221)$ showed the strongest facilitation effects for the High-Challenge group on episodic memory after the intervention. We thus focused first on the episodic memory construct. Episodic memory was assessed using three measures: the immediate and long-delay recall section of the modified Hopkins Verbal Learning Task (Brandt, 1991), and the Cambridge Neuropsychological Test Automated Battery (CANTAB) Verbal Recognition Memory Task (Robbins et al., 1994). Additionally, we examined changes in verbal fluency (Bechtoldt et al., 1962) at pretest and posttest. In the verbal fluency task, participants generated as many unique words as possible that started with a specific letter $(\mathrm{F}, \mathrm{A}$, and $\mathrm{S})$ in 60 seconds. This task was of particular interest because it shared several components with the fMRI task. Both tasks 1) required retrieval of semantic information from memory, 2) placed demands on working memory, and 3) have been shown to activate similar brain regions including left inferior frontal gyrus (Cabeza \& Nyberg, 2000; Collette \& Van der Linden, 2002).

\subsubsection{MRI acquisition}

All participants were scanned on a single 3T Philips Achieva scanner equipped with an 8-channel head coil. High-resolution anatomical images were collected with a structural high-resolution T1-weighted MP-RAGE sequence with 160 sagittal slices, $1 \times 1 \times 1 \mathrm{~mm}^{3}$;
$256 \times 256 \times 160$ matrix, $\mathrm{TR}=8.18 \mathrm{~ms}, \mathrm{TE}=3.76 \mathrm{~ms}$, flip-angle $=12^{\circ}, F O V=220 \mathrm{~mm}$. BOLD fMRI data were acquired using a T2*-weighted echo-planar imaging sequence (with SENSE encoding) with 43 interleaved axial slices per volume acquired parallel to the AC-PC line, $64 \times 64 \times 43$ matrix $(3.4 \times$ $\left.3.4 \times 3.5 \mathrm{~mm}^{3}\right), \quad \mathrm{FOV}=220 \mathrm{~mm}, \quad \mathrm{TE}=25 \mathrm{~ms}, \quad \mathrm{TR}=$ $2 \mathrm{sec}, \mathrm{FA}=80^{\circ}$. Five dummy volumes were discarded at the beginning of the functional run to allow for $\mathrm{T} 1$ stabilization.

\subsection{MRI data analyses}

\subsubsection{Preprocessing}

Preprocessing was completed using Statistical Parametric Mapping software (SPM8: Wellcome Department of Cognitive Neurology, University College London, London, United Kingdom). Images were aligned to the first retained volume of the run to account for individual participant movement. Following this, the anatomical scans were segmented, skull-stripped, and then parameter estimates from the deformation and segmentation process were used to co-register and align the functional images to the structural image. The functional images from all three time points (pretest, posttest, one year follow-up) were then normalized to Montreal Neurological Imaging (MNI) space and resampled to $3 \mathrm{~mm}^{3}$ voxels. Quality of the normalization process was checked across participants and the three time points. Specifically, mean functional images were stacked and then visually inspected for across-participant spatial deviations or "jumps" near ventricles and around the edges of the skull. No large deviations were detected. Motion-corrected volumes were spatially smoothed using a Gaussian filter with a $6 \mathrm{~mm}$ full-width at half-maximum (FWHM) kernel. Additionally, Multivariate Exploratory Linear Optimized Decomposition into Independent Components (MELODIC; Beckmann \& Smith, 2004) was used to identify and remove obvious artifacts such as motion and physiological noise.

\subsubsection{First level analyses}

For each participant and time point, first level (participant) analyses were conducted using a voxelwise general linear model (GLM) that was high-pass filtered using $1 / 72 \mathrm{~Hz}$. This high-pass filter was chosen because blocked designs introduce brain signals of interest at lower frequencies than event-related designs, and using a high-pass filter at a frequency 
too high (i.e., $1 / 128 \mathrm{~Hz}$ ) can remove task-relevant signals of brain activity. For each time point, easy and hard blocks were modeled, including rigid body and rotation regressors as covariates, to obtain the relevant parameter estimates. For each person at each of the three time points, a brain modulation map was created by contrasting brain activity associated with the hard vs. easy condition. These contrasts were then used as the dependent measure for all second level analyses that assessed the effect of intervention group from pretest to posttest.

\subsubsection{Second level analyses}

These whole-brain analyses used a threshold set at $p<0.004$ and a cluster size of at least 29 contiguous voxels, resulting in a corrected threshold of $p<0.01$ as determined by AlphaSim (B.D. Ward). The primary fMRI analysis was conducted in a series of steps. In Step 1, we contrasted hard > easy at pretest separately for each group to examine the general pattern of modulation before the intervention. In Step 2, we assessed whether the Low-Challenge and HighChallenge groups differed in modulation at pretest by calculating an independent samples $t$-test of the brain modulation maps between the two groups. In Step 3, we assessed the impact of the intervention for each group by contrasting pretest and posttest modulation using a paired $t$-test. In a fourth step, we averaged the $t$-values from each significant cluster from each time point isolated in Step 3, and submitted them to a Group (Low Challenge, High Challenge) $\times$ Time $($ Pre, Post $)$ mixed ANOVA using SPSS statistical software package (Version 21; IMB Corp, 2012). All interactions surviving $p<0.05$ were considered significant.

Net effect sizes were calculated using the formula: $\left[\left(T_{\mathrm{h}}{ }^{\text {post }}-T_{1}{ }^{\text {post }}\right)-\left(T_{\mathrm{h}}{ }^{\text {pre }}-T_{1}{ }^{\text {pre }}\right)\right] / \mathrm{s}^{\text {pre }}$, where $\mathrm{s}^{\text {pre }}$ is the standard deviation at pretest across all participants, $T_{\mathrm{h}}{ }^{\text {pre }}$ and $T_{\mathrm{h}}{ }^{\text {post }}$ represent pretest and posttest $t$-values for the High-Challenge group, and $T_{1}{ }^{\text {pre }}$ and $T_{1}{ }^{\text {post }}$ represent pretest and posttest $t$-values for the Low-Challenge group. As guided by Ferguson (2009), we set 0.41 as a minimum level for small effect sizes, 1.15 for moderate effects sizes, and 2.70 for large effect sizes. Finally, as in Ball et al. (2002) and Park et al. (2014), percent reliable change resulting from the intervention was calculated using the formula: $\left(T_{\mathrm{h}}{ }^{\text {post }}\right.$ $\left.T_{\mathrm{h}}{ }^{\text {pre }}\right) / \mathrm{s}^{\text {pre }} \geq \mathrm{s}^{\text {pre }} \sqrt{ }(1-\mathrm{R})$, where $\mathrm{R}$ is the reliability of each cluster of activity obtained from the Low-Challenge group.

\section{Results}

\subsection{Participant demographics}

The demographics for the High-Challenge and LowChallenge groups did not differ significantly in age, sex, years of education, MMSE, nor adherence hours to the intervention (all $p$ 's $>0.78$; see Table 1). The groups also did not differ from each other on these measures in the one year follow-up sample $(N=26)$, all $p$ 's $>0.33$. We also tested whether extra-experimental activities that were beyond our control differed between the two groups. To do this, participants filled out a 70-item questionnaire each week to assess the amount of outside activities (e.g., "This week did you: Prepare a meal? Drive a car? Take care of one or more pets? Play a musical instrument? Engage in exercise activities?). A 2 (Group: high challenge, low challenge) $\times$ Time (first week, last week) ANOVA on the amount of activities each week revealed only a main effect of Time $(p=0.037)$ such that outside activities decreased for both groups from the beginning to the end of the intervention. Thus, any differences between the two groups can most likely be attributed to the intervention manipulation rather than uncontrolled, extra-experimental activities.

\subsection{Scanner task performance}

Mixed analyses of variance (ANOVAs) were conducted on response times (Table 2). A Group (Low Challenge, High Challenge) $\times$ Item Type (Easy, Hard $) \times$ Time (Pretest, Posttest) ANOVA revealed only a main effect of Item Type, $F(1,37)=346.96$, $M S E=7.72, p<0.001$, confirming that the harder words required longer processing than easy words. No other effects were significant (all $p$ 's $>0.10$ ). Measures

Table 2

Response times for fMRI task as a function of group, difficulty, and time point

\begin{tabular}{lcc}
\hline & Low Challenge & High Challenge \\
\hline Easy RT & & \\
Pretest & $939.75(30.73)$ & $999.22(23.77)$ \\
Posttest & $964.31(34.65)$ & $982.85(27.13)$ \\
Hard RT & & \\
Pretest & $1186.41(43.51)$ & $1255.78(26.18)$ \\
Posttest & $1221.91(49.59)$ & $1287.78(28.30)$ \\
\hline
\end{tabular}

Notes. $1 \mathrm{Yr}=$ One-year follow up, RT = response times (in $\mathrm{ms}$ ). Accuracy data was only available for the easy condition. Standard Error of the Mean is in parentheses. 
of accuracy on the semantic judgment task were not analyzed because a) accuracy was near ceiling on easy blocks (means $>0.96$ ), and b) the harder words often were ambiguous and so did not have a single correct answer (e.g., sponge could be living or non-living).

\subsection{Engagement-related effects on modulation of brain activity}

As described earlier, the dependent variable for each participant was the whole-brain contrast of hard versus easy conditions at pretest and posttest, which provided a measure of modulation of brain activity to increasing task demands. The modulation data at pretest for each group are displayed in Fig. 1A and B. Overall participants showed increased modulation of brain activity to difficulty in prefrontal, lateral temporal, and some parietal regions consistent with modulation maps reported by Kennedy et al. (2015) using the same task. We then asssessed group differences at pretest by conducting an independent samples $t$-test between the High-Challenge and Low-Challenge groups. This analysis revealed only one significant cluster that differed at pretest- the left rolandic operculum (Low Challenge $>$ High Challenge). This region was not implicated in any further analyses and so will not be discussed further. We conclude that overall modulation was largely equivalent between groups at pretest.

Figure $1 \mathrm{C}$ and $\mathrm{D}$ display the posttest data. To examine change in the ability to modulate brain activity resulting from the intervention, we contrasted posttest with pretest in the High-Challenge group and in the Low-Challenge group (High-Challenge: Fig. $\mathrm{C}$ minus
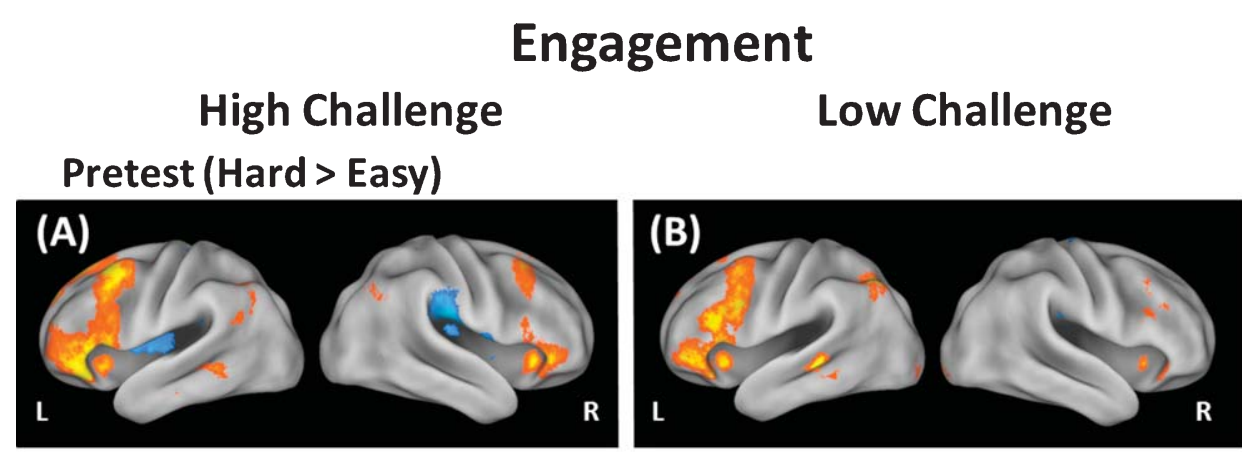

\section{Posttest (Hard > Easy)}
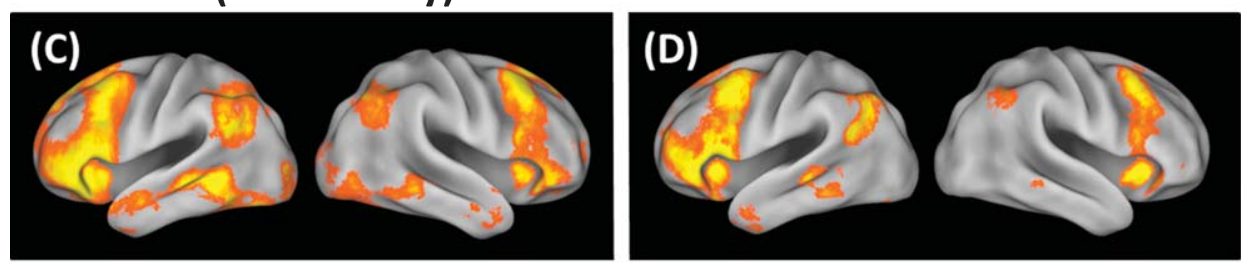

\section{Posttest > Pretest}

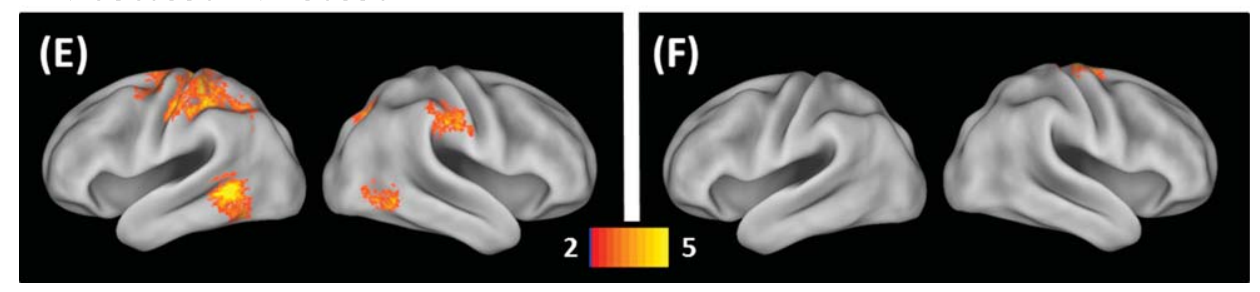

Fig. 1. Modulation of brain activity was measured at easy and hard levels of difficulty for the High-Challenge group (left) and Low-Challenge group (right). The top panels (A \& B) show modulation at pretest, the middle panels (C \& D) show modulation at posttest, and the bottom panels (E \& F) show modulation differences between pretest and posttest. The High-Challenge group exhibited increases in modulation of brain activity following the intervention in frontal, temporal, and parietal brain regions. In contrast, the Low-Challenge group only showed increases in modulation in one cluster (precentral gyrus). 
A; Low-Challenge: Fig. D minus B). The results of the contrasts are shown in Fig. 1E (High-Challenge), Fig. 1F (Low-Challenge), and Table 4. The HighChallenge group yielded 11 significant regions that showed increases in modulation including frontal, temporal, and parietal cortex. The same contrast for the Low-Challenge group resulted in only one cluster of increased modulation in right precentral gyrus. To understand how these clusters were affected by the intervention (as opposed to time or expectancy effects), we subjected each cluster to a Group $\times$ Time ANOVA in each of the 11 clusters. We found a significant interaction in five of the 12 clusters (see Table 4). All five significant interactions resulted from larger increases in modulation for the High-Challenge compared with the Low-Challenge group. The significant interactions are depicted in the left panel of Fig. 2 and occurred in left intraparietal sulcus, left middle temporal gyrus, right inferior temporal gyrus, left mid cingulate gyrus, and right precuneus. The temporal regions (left middle temporal and right inferior temporal) that showed engagement-related increases in modulation have been implicated in conceptual/object processing (e.g., Binder et al., 2009; Haxby et al., 2001). The frontal and parietal regions (mid cingulate and left intraparietal sulcus) have been suggested to play a role in attention and controlled processing (e.g., Cabeza et al., 2003; Corbetta \& Shulman, 2002). We note that the interactions took two forms. The first form in the left intraparietal sulcus and left middle temporal gyrus was characterized by a positive increase
Table 3

Behavioral performance on episodic memory and verbal fluency as a function of group and time point

\begin{tabular}{lcc}
\hline & Low Challenge & High Challenge \\
\hline CANTAB Verbal Recall Memory & & \\
$\quad$ Pretest & $7.00(0.32)$ & $7.39(0.28)$ \\
Posttest & $7.56(0.43)$ & $8.83(0.37)$ \\
$\quad$ Difference & $0.56(0.29)$ & $1.44(0.32)$ \\
Verbal Fluency & & \\
Pretest & $38.94(2.44)$ & $39.52(2.63)$ \\
Posttest & $43.56(2.39)$ & $43.17(2.67)$ \\
Difference & $4.63(1.10)$ & $3.65(1.09)$ \\
\hline
\end{tabular}

Note. Standard Error of the Mean is in parentheses.

in modulation of brain activity to difficulty at pretest, followed by additional increases in modulation from pretest to posttest, but only for the High-Challenge group. The second form of interactions were found in the other three clusters (left mid cingulate gyrus, right precuneus, and right inferior temporal gyrus) and showed little evidence of modulation to difficulty at pretest, but exhibited increased modulation after the engagement in the High-Challenge group only. We note that in these latter clusters, the High-Challenge group showed negative modulation effects at pretest (easy $>$ hard), thus potentially playing a role in the significant interaction.

Net effect sizes associated with the significant regions are reported in Table 4 and ranged from small to moderate effect sizes. Figure 2 presents estimates of reliable change for the five significant interactions and shows that $57-78 \%$ of participants evidenced a reliable

Table 4

Engagement-related increases in brain activity

\begin{tabular}{|c|c|c|c|c|c|c|c|c|}
\hline \multirow{2}{*}{$\begin{array}{l}\text { Region } \\
\text { High Challenge (Posttest > Pretest }\end{array}$} & \multicolumn{3}{|c|}{$\begin{array}{l}\text { MNI Coordinates } \\
(\mathrm{x}, \mathrm{y}, \mathrm{z})\end{array}$} & \multirow[t]{2}{*}{ BA } & \multirow[t]{2}{*}{$\begin{array}{l}\text { Cluster } \\
\text { Size }\end{array}$} & \multirow[t]{2}{*}{$\begin{array}{l}\text { Net Effect } \\
\text { Size }\end{array}$} & \multirow[t]{2}{*}{$\begin{array}{c}\% \text { Reliable } \\
\text { Change }\end{array}$} & \multirow[t]{2}{*}{$\begin{array}{c}\text { Group } \times \text { Time }(\text { Pre-Post }) \\
\text { Interaction } p \text {-value } \\
\end{array}$} \\
\hline & & & & & & & & \\
\hline L Mid Cingulate Gyrus & -6 & -4 & 30 & 24 & 47 & 1.73 & 0.78 & 0.00 \\
\hline $\mathrm{R}$ Precuneus & 20 & -62 & 44 & 7 & 34 & 1.09 & 0.65 & 0.01 \\
\hline L Intraparietal Sulcus & -38 & -59 & 51 & 40 & 29 & 0.84 & 0.26 & 0.01 \\
\hline R Inferior Temporal Gyrus & 56 & -64 & -7 & 37 & 29 & 1.02 & 0.57 & 0.02 \\
\hline L Middle Temporal Gyrus & -55 & -53 & -4 & 21 & 98 & 0.91 & 0.65 & 0.02 \\
\hline L Postcentral (Ventral) Gyrus & -40 & -32 & 51 & $2 / 40$ & 168 & 0.81 & 0.52 & 0.10 \\
\hline R SupraMarginal Gyrus & 56 & -25 & 40 & $2 / 3 / 4$ & 38 & 0.69 & 0.57 & 0.10 \\
\hline L Putamen & -26 & -1 & -5 & - & 47 & 0.73 & 0.65 & 0.11 \\
\hline L Precentral Gyrus & -27 & -12 & 66 & 6 & 63 & 0.65 & 0.65 & 0.13 \\
\hline L Postcentral (Dorsal) Gyrus & -16 & -34 & 69 & $3 / 4$ & 43 & 0.60 & 0.61 & 0.17 \\
\hline L Thalamus & -4 & -10 & 5 & - & 40 & 0.64 & 0.52 & 0.21 \\
\hline \multicolumn{9}{|l|}{ Low Challenge (Posttest > Pretest) } \\
\hline R Precentral Gyrus & 26 & -17 & 73 & 6 & 41 & 0.65 & 0.43 & 0.18 \\
\hline
\end{tabular}

Notes. Regions are sorted according to interaction significance. $\mathrm{L}=\mathrm{Left}, \mathrm{R}=\mathrm{Right}, \mathrm{BA}=$ Approximate Brodmann Area. Only increases in brain activity were significant using a whole brain threshold of $p<0.01$, corrected. Post-hoc Group $\times$ Time interactions were considered significant at $p<0.05$. 


\section{Bar Plots}
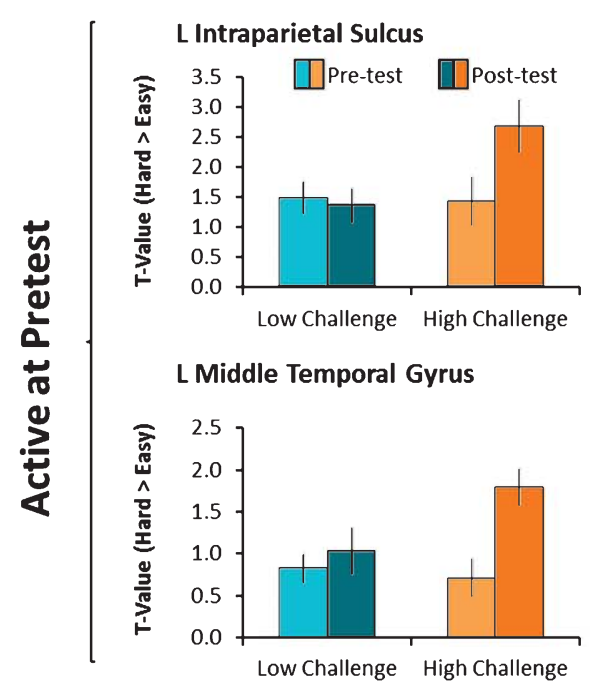

R Inferior Temporal Gyrus

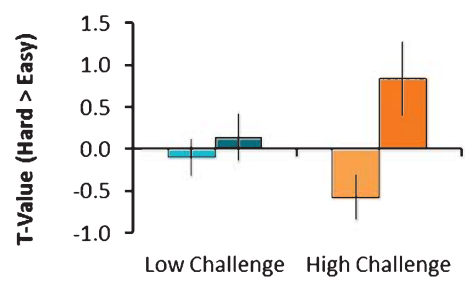

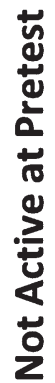

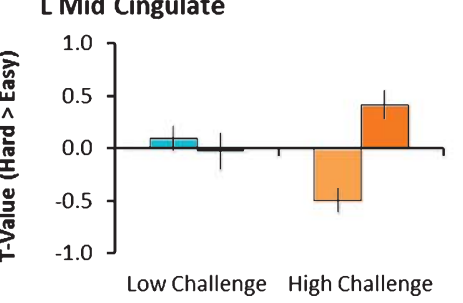

R Precuneus

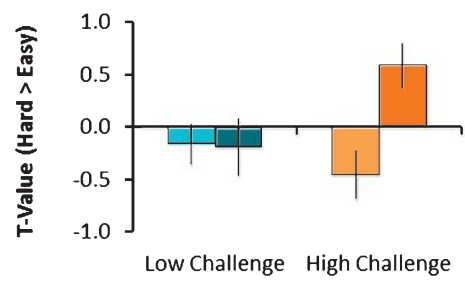

Individual Gain Scores
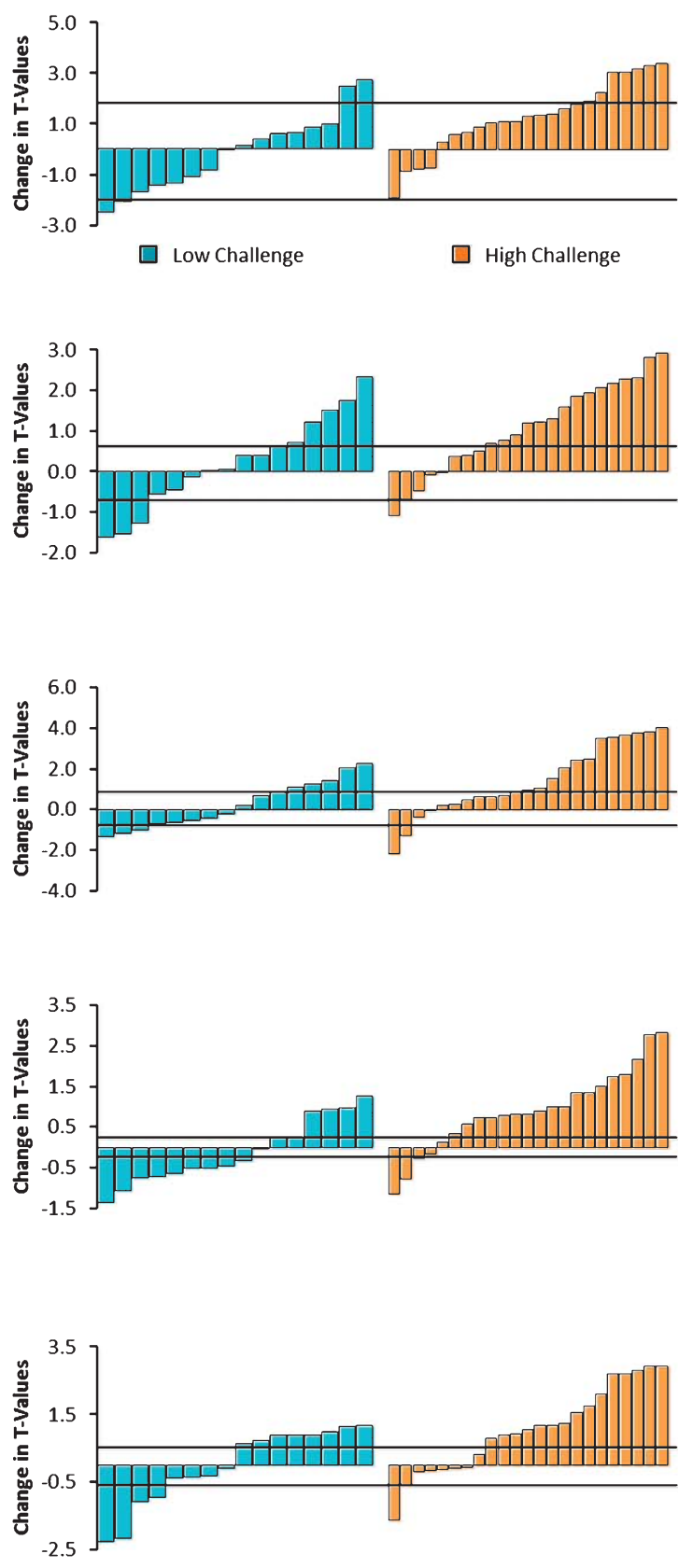

Fig. 2. Modulation of brain activity was averaged across voxels in clusters showing a significant Group $\times$ Time interaction and plotted in the left panel. As can be seen in the bar plots on the left, the Low-Challenge group (blue) showed no differences in brain modulation from pre to posttest, but the High Challenge group (orange) showed increases in brain modulation from pre to posttest. On the right, individual gain scores in modulation of brain activity are shown. While individuals varied in the degree to which they showed increases or decreases in modulation of brain activity, a greater proportion of individuals in the High-Challenge group showed engagement-related increases. The horizontal black bars represent the standard error of measurement (+1 SEM and -1 SEM). 
increase in modulation of brain activity due to highchallenge engagement. One exception to this finding was in the left intraparietal sulcus, which showed only a $26 \%$ reliable increase in brain activity due to large variability of brain activity in this region.

\subsection{Locus of modulation effects}

The significant modulation differences between high-challenge engagement and low-challenge engagement could be due to 1) decreases in brain activity in the easy condition (i.e., more efficient processing), 2) increases in brain activity in the hard condition (i.e., greater recruitment of neural resources), or 3 ) a combination of both. To test this, we conducted two separate ANOVAs. In the first ANOVA, we analyzed differences in brain activity from pretest to postest in the easy trials versus fixation, including the variables of Group (High Challenge, Low Challenge), Region (L IPS, L MTG, R ITG, R Precunues, L Cingulate), and Time (Pretest, Posttest). We conducted a similar ANOVA on only the hard items as well. For the easy condition, we found a Group $\times$ Time interaction, $F(1,37)=4.11$, $M S E=2.79, p=0.05$, but no 3-way interaction, $F$ $(4,148)=0.46, M S E=0.87, p=0.76$. The two-way interaction occurred because the High-Challenge group showed a greater decrease from pretest to posttest than the Low-Challenge group (see Fig. 3). This pattern of decreased activity was similar across the five regions. In contrast, the same analysis for the hard condition showed no interactions (all $p$ 's $>0.72$ ), suggesting that the increased modulation observed in the original analysis was a result of the HighChallenge group utilizing fewer neural resources in the easy condition after the intervention.

In sum, separate analysis of the easy and hard condition support the idea that increased modulation in the High-Challenge group was due to the easy condition requiring less neural resource after prolonged participation in high-challenge engagement. Because the easy task required a simple semantic judgment, the findings suggest that the experience of sustained highchallenge engagement led to more fluent retrieval of semantic information.

\subsection{Relationship of modulation of brain activity to adherence to the program}

Participants were free to spend more than the required 15 hours per week in the Synapse space and many did. To determine whether variability in time spent in the Synapse environment translated to greater modulation effects, we correlated the number of hours spent in the program with modulation change scores for the five significant clusters as a function of group
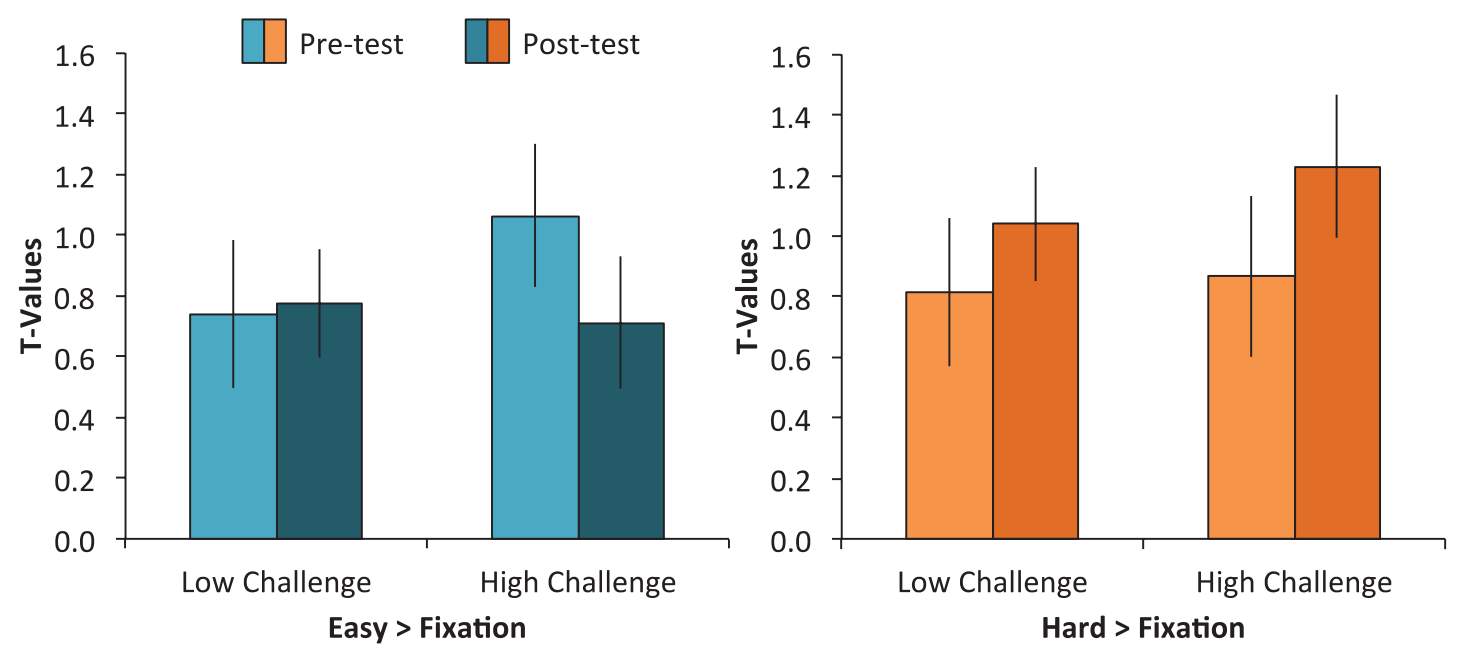

Fig. 3. Modulation of brain activity (hard>easy) was further broken down within each condition and engagement group averaged across the five clusters that showed significant engagement effects. The left panel shows mean brain activity in both engagement groups for the easy $>$ fixation contrast and the right panel shows mean brain activity in both engagement groups for the hard $>$ fixation contrast. A Group $\times$ Time interaction for the easy condition, but not the hard condition, suggests that brain activity decreased from pretest to posttest selectively in the High-Challenge group. 
Table 5

Correlations with changes in brain activity, adherence hours, verbal fluency, and age

\begin{tabular}{lccl}
\hline Region & $\begin{array}{c}\text { Adherence } \\
\text { Hours }\end{array}$ & $\begin{array}{c}\text { Verbal } \\
\text { Fluency Gains }\end{array}$ & Age \\
\hline High Challenge & & & \\
L Mid Cingulate Gyrus & $0.43^{*}$ & 0.32 & 0.22 \\
R Precuneus & $0.53^{* *}$ & $0.38^{\dagger}$ & $0.66^{* *}$ \\
L Intraparietal Sulcus & $0.37^{\dagger}$ & 0.18 & $0.50^{*}$ \\
R Inferior Temporal Gyrus & $0.64^{* *}$ & $0.43^{*}$ & $0.54^{* *}$ \\
L Middle Temporal Gyrus & $0.37^{\dagger}$ & $0.35^{\dagger}$ & $0.44^{*}$ \\
Low Challenge & & & \\
L Mid Cingulate Gyrus & -0.19 & $-0.43^{\dagger}$ & 0.39 \\
R Precuneus & -0.13 & -0.02 & 0.28 \\
L Intraparietal Sulcus & -0.07 & -0.31 & 0.28 \\
R Inferior Temporal Gyrus & 0.01 & -0.25 & 0.30 \\
L Middle Temporal Gyrus & 0.34 & 0.08 & 0.20 \\
\hline
\end{tabular}

Notes. ${ }^{\dagger} p<0.10,{ }^{*} p<0.05,{ }^{* *} p<0.01, \mathrm{~L}=$ left, $\mathrm{R}=$ right.

(Table 5). One outlier from each group was removed from the analysis with adherence hours because their hours were greater than 2.5 standard deviations from the mean. In the High-Challenge group, greater modulation changes in three clusters (left mid cingulate gyrus, right precuneus, and right inferior temporal gyrus) were associated with more adherence hours ( $r$ 's $=0.43-0.64, p$ 's $<0.05)$. Similarly, greater changes in modulation in two clusters (left intraparietal sulcus and left middle temporal gyrus) were associated with more adherence hours ( $r$ 's $=0.37, p$ 's $<0.10)$. In the Low-Challenge group, none of the correlations were significant and many were in the negative direction (all $p$ 's $>0.10$ ). This result suggests that individuals who spent more time learning new skills were more likely to show increased modulation of brain activity.

\subsection{Relationship of modulation of brain activity to cognition}

An analysis of the constructs from the cognitive battery (processing speed, episodic memory, mental control, and visuospatial processing) as a function of group and time yielded no significant effects. Because Park et al. (2014) found an effect on episodic memory, we analyzed the 3 component tasks of the episodic memory constructed, which yielded a marginal interaction for the individual CANTAB Verbal Recall Task, $F(1,37)=3.71, p=0.06$, that was in the appropriate direction (High Challenge gains $>$ Low Challenge gains; see Table 3 ). These results are not surprising given the considerably smaller sample size compared to the original study ( $N=221$ vs. $N=39$; Park et al., 2014) and are not the focus of the present paper. Nevertheless, these findings do not preclude the possibility that individual differences in modulation gains would be correlated with individual differences in cognition. Thus, we correlated changes in modulation of brain activity with changes in episodic memory. We also examined the relationship between modulation and verbal fluency because this task shared several components with the fMRI task. For episodic memory, we found no significant correlations in either group. However, as shown in Table 5, we did find that increases in verbal fluency in the HighChallenge group were significantly correlated with increased modulation in the right inferior temporal gyrus $(r=0.43, p<0.05)$, and two clusters (right precuneus and left middle temporal gyrus) showed marginal correlations ( $r$ 's $=0.35-0.38, p$ 's $<0.10)$. In the Low-Challenge group, all the correlations were near zero or negative (all $p$ 's $>0.05$ ).

\subsection{Relationship of modulation of brain activity to age}

To further investigate what characteristics were associated with greater modulation of brain activity, we correlated pretest to posttest changes in modulation of brain activity with age at pretest. In the High-Challenge group, changes in modulation were positively correlated with age for four of the five significant clusters (all but mid cingulate gyrus; $r$ 's $=0.44-0.66, p$ 's $<0.05$ ). In the Low-Challenge group, changes in modulation did not vary by age (all $p$ 's $>0.10$ ). These results show that the older the individuals that entered into the program, the greater the brain changes.

These positive correlations might be due to the fact that the youngest old had the greatest modulation at pretest, and thus had less room to improve compared to the oldest adults. To test this idea, we examined the relationship of modulation at pretest in the HighChallenge group. As expected, there were significant negative correlations in the High-Challenge group (all but mid cingulate gyrus; $r$ 's $=-0.45--0.60, p$ 's $<0.05$ ), consistent with the idea that the oldest participants had the most room to improve.

However, we recognize that another explanation is that these age effects are simply due to "regression towards the mean". If this were the case, one would expect the Low-Challenge participants with low 
modulation at pretest would show greater gains at posttest (i.e., a negative correlation between pretest level of modulation and gains in modulation). Correlations in the Low-Challenge group were not significant for three of the five clusters (middle temporal gyrus, precuneus, and inferior temporal gyrus; all $p$ 's $>0.14)$, marginally negative in the mid cingulate gyrus $(\mathrm{r}(16)=-0.47, p=0.07)$, and significantly negative in the precuneus $(\mathrm{r}(16)=-0.69, p=0.003)$. Thus, while we cannot rule out the "regression toward the mean" explanation in all of the clusters, it appears as if this explanation is not tenable for many of them.
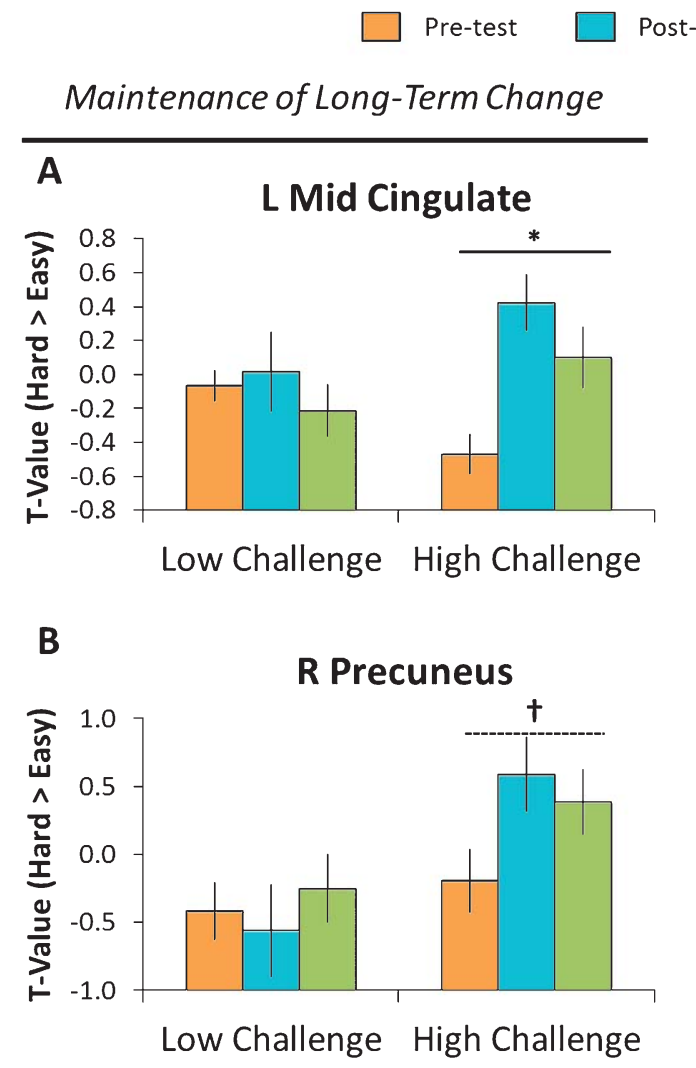

D
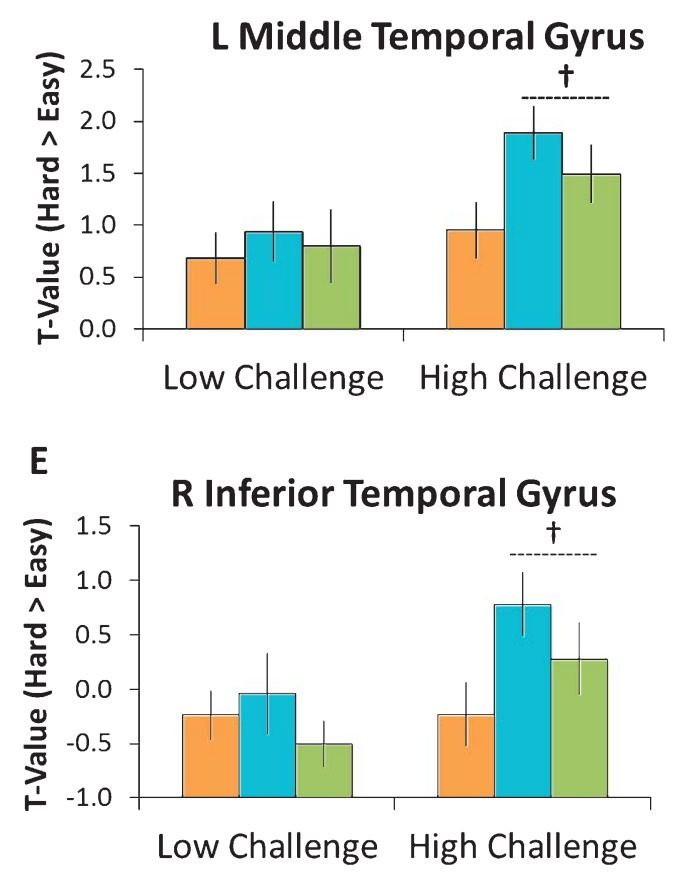

Fig. 4. One year following the intervention, a subsample of participants $(N=26)$ was re-tested. Patterns of brain activity fell into two categories. The first category consisted of individuals that showed maintenance of long-term benefits as assessed by a difference from pretest to the one year follow-up, but no difference from posttest to the one year follow-up (left column). These regions included the left mid cingulate gyrus and the right precuneus. The second category consisted of individuals that showed intermediate long-term change as assessed by no difference from pretest to the one year follow-up, but a marginal difference from posttest to the one year follow-up (right column). These regions included the left intraparietal sulcus, the left middle temporal gyrus, and the right inferior temporal gyrus. ${ }^{\dagger} p<0.10,{ }^{*} p<0.05$. 


\subsection{Maintenance of engagement after one year}

To determine whether sustained and challenging engagement resulted in long-term brain changes, we conducted a 2 (Group: Low Challenge, High Challenge) $\times 3$ (Time: Pretest, Posttest, One year) ANOVA on the five significant clusters displayed in Fig. 2 and tested for for both linear and quadratic interactions across the three time intervals. Evidence for maintenance of the effects under ideal conditions would occur if the increases in modulation a) differed between pretest and one year, and b) did not differ between posttest and one year. The results for the five clusters are displayed in Fig. 4 and are discussed in turn.

As shown in Fig. 4A and B, the left mid cingulate gyrus and to a lesser extent the right precuneus revealed patterns consistent with maintenance of the engagement effects. Specifically, the mid cingulate gyrus exhibited a significant linear interaction, $F_{\text {linear }}$ $(1,24)=4.89, p=0.04$ that occurred because there was little change in the Low-Challenge group over the three time points, whereas the High-Challenge group showed increases in modulation of brain activity from pretest to one year, $t(15)=2.46, p=0.03$, and did not differ from posttest to one year $(p=0.14)$. A similar effect was observed for the right precuneus, but the interaction did not reach significance, $F_{\text {linear }}$ $(1,24)=0.90, p=0.35$. This region showed no difference over the three time points for the Low-Challenge group, whereas the High-Challenge group showed a marginally significant increase in modulation from pretest to one year, $t(15)=2.08, p=0.06$, and no difference from posttest to one year later $(p=0.54)$.

Evidence for less robust benefits resulting from the High-Challenge engagement occurred in the other three regions and are displayed in Fig. 4C-E. In these regions, there was a significant increase in only the High-Challenge group from pretest to one year, with marginal decreases in modulation of brain activity from posttest to one year follow-up. While this posttest to one-year difference was not significant-and thus suggestive of a maintenance of the intervention effects - these three regions also did not significantly differ between pretest and one year follow-up, limiting the conclusion that these increases were maintained. Specifically, the left intraparietal sulcus exhibited a significant quadratic interaction, $F_{\text {quadratic }}(1,24)=7.05$, $p=0.03$, that occurred because the Low-Challenge group showed slight decreases across the three time points, whereas the High-Challenge group showed a nonsignificant increase from pretest to one year, $t$ $(15)=1.15, p=0.27$, and marginally decreased from posttest to one year, $t(15)=1.99, p=0.07$. Both the left middle temporal gyrus and right inferior temporal gyrus showed a similar, but nonsignificant pattern $\left(F_{\text {quadratic }}(1,24)=1.78, p=0.20\right.$ and $F_{\text {quadratic }}$ (1, $24)=0.93, p=0.35$, respectively), evidencing no differences from pretest to one year, $p$ 's $>0.11$, and showing a marginal difference from posttest to one year, $t(15)=2.07, p=0.06$ and $t(15)=1.77, p=0.10$ for middle and inferior temporal gyri, respectively.

\section{Discussion}

The present findings provide some of the first experimental evidence that older adults who have a sustained engagement in a mentally-challenging environment show increased modulation of brain activity. This finding is consistent with the idea that interventions can restore levels of brain activity to a more youth-like state (Nyberg et al., 2012; Park \& Reuter-Lorenz, 2009). The engagement-related neural effects took the form of decreases in brain activity when making easy semantic judgments, and appear to at least partially endure for one year. Individual differences variables such as age, time spent in the engaged environment, and changes in verbal fluency were associated with stronger modulation effects. Of particular importance is the finding that participants who spent an equal amount of time in a low challenge, social environment did not show these same patterns, providing evidence that the modulation effects could be attributed to mental challenge and not to effects of socializing and/or other activities that required little new learning. The present results provide support for the "Use it or lose it" hypothesis of cognitive aging (e.g., Hertzog et al., 2009; Hultsch et al., 1999; Salthouse, 2006). We discuss major components of the findings below.

\subsection{Types of brain changes resulting from engagement}

There is convergent evidence from multiple studies indicating that older adults have a reduced capacity to modulate brain activity in response to increasing task demands (e.g., Kennedy et al., 2015; ReuterLorenz \& Cappell, 2008; Schneider-Garces et al., 2010). We reported five brain regions that showed significant improvement in modulatory capacity during a 
semantic judgment task after three months of sustained and high challenge engagement. The regions showing this pattern spanned two broad domains of cognition. The first set consisted of frontoparietal brain regions that are often implicated in attentional processing and executive function (e.g., Cabeza et al., 2003; Corbetta \& Shulman, 2002). Critically, these regions show significant age-related declines from young to middle-age and from middle-age to old age on the same semantic judgment task (Kennedy et al., 2015). Because modulation of these regions decline with age, the engagement-related increases in modulation provide evidence that engagement may have partially restored age-related declines in brain activity related to attentional processing and/or executive function.

The second set of brain regions that showed increased modulation included inferior temporal cortex-a region implicated in object processing and object recognition (e.g., Haxby et al., 2001)-and middle temporal cortex-a region associated with conceptual or semantic processing (e.g., Binder et al., 2009). The inferior and middle temporal regions also were isolated independently in the Kennedy et al. (2015) study as regions associated with agerelated decreases in modulation. Interestingly, the engagement-related modulation effects observed in left middle temporal gyrus were similar in location to one of the regions that showed increased modulation effects in a study explicitly designed to train semanticelaboration strategies in older adults (Kirchhoff et al., 2012). However, unlike training studies, the Synapse Project was unique in that no cognitive processes were explicitly trained. We suggest that the Synapse Project operated as a type of environmental support to reorient older individuals to activate and direct more processing resources externally toward their environment, thus facilitating attention and semantic elaboration beyond the Synapse environment.

The topography of the brain regions increased in the High-Challenge group is of considerable interest. A visual comparison of modulation at pretest (Fig. 1A and B) with the regions of modulation increases (Fig. 1E) suggest that three of the five clusters (right inferior temporal gyrus, left mid cingulate, and right precuneus) showing a Group $\times$ Time interaction were located in new brain regions that were not implicated as a function of task demands at pretest. At first glance, this finding appears to be consistent with the development of experience-based and distributed neural scaffolds (Park \& Reuter-Lorenz,
2009; Reuter-Lorenz \& Park, 2014). However, further investigation of the nature of these modulation effects suggests that the increases in modulation were due to decreases in brain activity during the easy condition rather than increases in brain activity during the hard condition. Thus, rather than recruiting new brain regions, older adults seem to be more efficient in how they utilize their existing neural resources. This finding might indicate that over time, older adults naturally develop neural scaffolds as a compensatory function, but that engagement in sustained and high-challenge tasks removes the need for such compensation. A different perspective on the impact of the engagement intervention comes from studies suggesting that older adults, compared with young adults, non-selectively recruit brain regions that interrupt or slow down cognitive processing (e.g., Logan et al., 2002). If this were the case, high-challenge engagement could reduce this non-selectivity. The important point is that either of these interpretations supports the idea that these age differences might be, at least partially, restored to more youth-like states through high-challenge engagement.

While some of the regions showing this increased neural efficiency (i.e., mid cingulate and precuneus) lasted up to one year later, many of the other regions began to return to their pretest levels of brain modulation. This apparent return to pretest levels could mean that engagement must continue over longer periods-perhaps even a lifetime-to maintain such modulation effects.

In contrast to the brain regions that showed increased modulation in new brain regions, two brain regions (left middle temporal gyrus and left intraparietal sulcus) exhibited significant modulation at pretest and showed further increases in modulation following engagement in the High-Challenge group. The left middle temporal gyrus is well-known for its role in the representation of verbal semantic information (e.g., Blumenfeld et al., 2006; Booth et al., 2002) and has even been suggested to be the principal storage site for information regarding objects and their attributes (e.g., Binder et al., 2009). Increased activity in temporal regions also has been shown to be correlated with more accurate category judgments, further supporting its importance in semantic processing (e.g., Chou et al., 2006). On the other hand, dorsal parietal activation such as in the intraparietal sulcus has been found on semantic tasks that vary in difficulty and has been suggested to play a role in attention and controlled processing rather than semantic processing per se (e.g., Binder et al., 2009; 
Sabsevitz et al., 2005). These effects may be additive; as attentional capacity is restored via the intervention, individuals would be better able to access or sustain semantic representations.

\subsection{Individual differences in brain changes resulting from engagement}

Shors (2014) reported that the more time and effort required for successful learning in animals, the more neurons were retained (i.e., less cell death) following neurogenesis. Generalizing this evidence to humans, we found a dose-response effect relating the amount of time spent in High-Challenge engagement to increases in brain modulation capacity. While not all individuals showed equal increases in modulation, this finding suggests that extending the length of the Synapse intervention (assuming that a high level of interest could be maintained) would have led to more participants benefitting from the program.

In addition to time spent in the program, we found evidence that the oldest individuals in the HighChallenge group showed the greatest increases in brain modulation. This may be because the oldest individuals were the most disengaged at pretest, resulting in larger effects than younger participants who had more social ties and opportunities for engagement. Alternatively, the oldest individuals could have had the most neural insults (e.g., brain shrinkage, dopamine depletion), and therefore were the most in need of fortifying neural resources and maintaining neural efficiency. Consistent with both of these ideas, we found that at pretest, the oldest individuals had the lowest level of neural modulation (see also, Kennedy et al., 2015).

Were these increases in modulation of brain activity truly beneficial? There is some debate as to whether increases in modulation are facilitative of cognition or a sign of mental deterioration. Evidence from this study points to a facilitative effect. First, the engagement intervention in the larger sample showed robust increases in memory (Park et al., 2014), which was marginally retained in the present study. Second, people in the High-Challenge group that showed greater increases in brain modulation also showed greater increases in verbal fluency performance. The increase in fluency was associated with brain regions isolated by the contrast of posttest $>$ pretest including the inferior temporal cortex-implicated in semantic/conceptual processes. Lastly, we found that the nature of the modulation effects were due to decreases in brain activity in the easy condition across the five significant clusters, suggestive of a more efficient use of neural resources.

\subsection{Alternative explanations}

Could the engagement-related increases in modulation of brain activity be due to factors other than a facilitation of cognitive processing? We considered that there could be a generalized compliance effect such that the High-Challenge group was willing to invest more effort than the Low-Challenge group, or perhaps the High-Challenge group ruminated in more depth during the hard judgment condition, or even that the High-Challenge group exhibited less efficient brain activity in the harder condition. While analyses of the modulation effects (hard > easy) cannot distinguish between such alternatives, the follow-up analyses of brain activity separately during each condition compared with baseline provided more information about the plausibility of these hypotheses. Specifically, all of the aforementioned alternative explanations would have predicted that the nature of the modulation effects stemmed from increased brain activity either in the hard condition alone, or in both the easy and the hard condition. However, we found that the nature of the effects stemmed from decreases in brain activity in the easy condition, and no significant engagement effects in the hard condition, thus ruling out those alternatives.

Another alternative explanation related specifically to the decreases in brain activity is that high-challenge engagement improved processing fluency or conceptual repetition priming. While behavioral evidence suggests that priming is spared in older adults, younger adults consistently show greater decreases in brain activity (i.e., repetition suppression) during priming than older adults (e.g., Ballesteros et al., 2013; Bergerbest et al., 2009). Therefore, the current study may have improved neural repetition suppression in older adults. While possible, there are several reasons why we believe this interpretation is less likely. First, response times did not evidence repetition priming from pretest to posttest for either engagement group despite evidence that some types of priming can last weeks (Cave, 1997) or even years (Mitchell, 2006). Second, repetition suppression should have occurred for both easy and hard conditions, but this pattern was not exhibited. 


\subsection{Limitations and future research}

We recognize that the interpretation of engagement as a mechanism for restoring deficits in semantic elaboration that occurs with age is a working hypothesis. The small sample made it difficult to replicate the episodic memory gains reported in Park et al. (2014) and we did not have an explicit measure of elaboration or a battery of semantic processing measures, which are limiting factors. Nevertheless, we found increased modulation of brain activity resulting from mental challenge that occurred in brain regions associated with attention and semantic processing. The inclusion of the social and placebo control group provides little doubt that the isolated brain differences can be attributed to sustained mental effort, as the amount of on-task time was nearly identical across groups. Other evidence reported in Park et al. (2014) indicated that the High-Challenge group did not differ from the Low-Challenge group in terms of subjective beliefs about their memory function, suggesting that demand characteristics also cannot explain the results. In future work, however, it would be useful to thoroughly probe demand characteristics at pretest and posttest and also to collect data after the intervention on the magnitude of engaged and cognitively-challenging behaviors in which the individuals participated. A finding of heightened post-engagement activities that were mentally effortful for High-Challenge compared to Low-Challenge participants would strengthen an understanding of mechanisms accounting for neural efficiency effects, especially those that endured.

\section{Conclusion}

The findings provide initial experimental support that engaging in challenging tasks for a sustained period of time increases neural efficiency in brain regions associated with attention and semantic processing, potentially enduring for relatively prolonged periods. We suggest that the mechanism through which engagement effects operate is via a restoration of brain activity to more youth-like states, thus facilitating the efficiency of neural resources that is a direct consequence of participation in a demanding learning environment. The findings superficially confirm the familiar adage regarding cognitive aging of "Use it or lose it." Although there is much more to be learned, we are cautiously optimistic with respect to the possibility that age-related cognitive declines can be slowed or even partially restored if individuals are exposed to sustained, mentally challenging experiences.

\section{Acknowledgments}

This research was supported by two National Institute on Aging grants to D. C. Park. Both were titled "Active Interventions for the Aging Mind": Grant 5R01AG026589 and American Recovery and Reinvestment Act (ARRA) Supplement 3R01AG026589-03S1. The authors thank Linda Drew, Jennifer Lodi-Smith, Carol Bardhoff, Katie Berglund, Michael Blackwell, Blair Flicker, Kim Flicker, Janice Gebhard, Mindi Kalosis, Kristen Kennedy, Anna Mortensen, Jenny Rieck, Karen Rodrigue, Linda Sides, Prassana Tamil, and Marcia Wood for assistance with this project.

\section{References}

Ball, K., Berch, D.B., Helmers, K.F., Jobe, J.B., Leveck, M.D., Marsiske, M., Morris, J.N., Rebok, G.W., Smith, D.M., Tennstedt, S.L., Unverzagt, F.W., \& Willis, S.L. (2002). Effects of cognitive training interventions with older adults: A randomized controlled trial. JAMA, 288(18), 2271-2281.

Ballesteros, S., Bischof, G.N., Goh, J.O., \& Park, D.C. (2013). Neural correlates of conceptual object priming in young and older adults: An event-related fMRI study. Neurobiol Aging, 34(4), 1254-1264.

Bechtoldt, H.P., Fogel, M.L., \& Benton, A.L. (1962). An Application of Factor-Analysis in Neuropsychology. Psychol Rec, 12(2), 147-156.

Beckmann, C.F., \& Smith, S.M. (2004). Probabilistic independent component analysis for functional magnetic resonance imaging. IEEE Trans Med Imaging, 23(2), 137-152.

Bergerbest, D., Gabrieli, J.D.E., Whitfield-Gabrieli, S., Kim, H., Stebbins, G.T., Bennett, D.A., \& Fleischman, D.A. (2009). Age-associated reduction of asymmetry in prefrontal function and preservation of conceptual repetition priming. Neuroimage, 45(1), 237-246.

Binder, J.R., Desai, R.H., Graves, W.W., \& Conant, L.L. (2009). Where is the semantic system? A critical review and metaanalysis of 120 functional neuroimaging studies. Cereb Cortex, 19(12), 2767-2796.

Blumenfeld, H.K., Booth, J.R., \& Burman, D.D. (2006). Differential prefrontal-temporal neural correlates of semantic processing in children. Brain Lang, 99(3), 226-235.

Booth, J.R., Burman, D.D., Meyer, J.R., Gitelman, D.R., Parrish, T.B., \& Mesulam, M.M. (2002). Modality independence of word comprehension. Hum Brain Mapp, 16(4), 251-261. 
Brandt, J. (1991). The hopkins verbal learning test: Development of a new memory test with six equivalent forms. Clin Neuropsychol, 5(2), 125-142.

Buitenweg, J.I., Murre, J.M., \& Ridderinkhof, K.R. (2012). Brain training in progress: A review of trainability in healthy seniors. Front Hum Neurosci, 6, 183.

Cabeza, R., Dolcos, F., Prince, S.E., Rice, H.J., Weissman, D.H., \& Nyberg, L. (2003). Attention-related activity during episodic memory retrieval: A cross-function fMRI study. Neuropsychologia, 41(3), 390-399.

Cabeza, R., \& Nyberg, L. (2000). Imaging cognition II: An empirical review of 275 PET and fMRI studies. J Cogn Neurosci, 12(1), $1-47$.

Carlson, M.C., Erickson, K.I., Kramer, A.F., Voss, M.W., Bolea, N., Mielke, M., McGill, S., Rebok, G.W., Seeman, T., \& Fried, L.P. (2009). Evidence for neurocognitive plasticity in at-risk older adults: The experience corps program. J Gerontol A-Biol, 64(12), 1275-1282.

Carlson, M.C., Saczynski, J.S., Rebok, G.W., Seeman, T., Glass, T.A., McGill, S., Tielsch, J., Frick, K., Hill, J., \& Fried, L.P. (2008). Exploring the effects of an "everyday" activity program on executive function and memory in older adults: Experience Corps. Gerontologist, 48(6), 793-801.

Cave, C.B. (1997). Very long-lasting priming in picture naming. Psychol Sci, 8(4), 322-325.

Chou, T.L., Booth, J.R., Bitan, T., Burman, D.D., Bigio, J.D., Cone, N.E., Lu, D., \& Cao, F. (2006). Developmental and skill effects on the neural correlates of semantic processing to visually presented words. Hum Brain Mapp, 27(11), 915-924.

Collette, F., \& Van der Linden, M. (2002). Brain imaging of the central executive component of working memory. Neurosci Biobehav Rev, 26(2), 105-125.

Corbetta, M., \& Shulman, G.L. (2002). Control of goal-directed and stimulus-driven attention in the brain. Nat Rev Neurosci, 3(3), 201-215.

Craik, F.I.M., \& Byrd, M. (1982). Aging and Cognitive Deficits. In F.I.M. Craik \& S. Trehub (Eds.), Aging and Cognitive Processes (Vol. 8, pp. 191-211). Springer US.

Craik, F.I.M., \& Jennings, J.M. (1992). Human memory. In F.I.M.C.T.A. Salthouse (Ed.), The handbook of aging and cognition (pp. 51-110). Hillsdale, NJ, England: Lawrence Erlbaum Associates, Inc.

Ferguson, C.J. (2009). An effect size primer: A guide for clinicians and researchers. Prof Psychol Res Pr, 40(5), 532-538.

Folstein, M.F., Folstein, S.E., \& McHugh, P.R. (1975). "Mini-mental state": A practical method for grading the cognitive state of patients for the clinician. J Psychiatr Res, 12(3), 189-198.

Grady, C. (2012). The cognitive neuroscience of ageing. Nat Rev Neurosci, 13(7), 491-505.

Greenwood, P.M. (2007). Functional plasticity in cognitive aging: Review and hypothesis. Neuropsychology, 21(6), 657-673.

Haxby, J.V., Gobbini, M.I., Furey, M.L., Ishai, A., Schouten, J.L., \& Pietrini, P. (2001). Distributed and overlapping representations of faces and objects in ventral temporal cortex. Science, 293(5539), 2425-2430.
Hertzog, C., Kramer, A.F., Wilson, R.S., \& Lindenberger, U. (2009). Enrichment effects on adult cognitive development. Perspect Psychol Sci, 9(1), 1-65.

Hultsch, D.F., Hertzog, C., Small, B.J., \& Dixon, R.A. (1999). Use it or lose it: Engaged lifestyle as a buffer of cognitive decline in aging? Psychol Aging, 14(2), 245-263.

Kennedy, K.M., Rodrigue, K.M., Bischof, G.N., Hebrank, A.C., Reuter-Lorenz, P.A., \& Park, D.C. (2015). Age trajectories of functional activation under conditions of low and high processing demands: An adult lifespan fMRI study of the aging brain. Neuroimage, 104, 21-34.

Kirchhoff, B.A., Anderson, B.A., Barch, D.M., \& Jacoby, L.L. (2012). Cognitive and neural effects of semantic encoding strategy training in older adults. Cereb Cortex, 22(4), 788-799.

Landau, S.M., Marks, S.M., Mormino, E.C., Rabinovici, G.D., Oh, H., O’Neil, J.P., Wilson, R.S., \& Jagust, W.J. (2012). Association of lifetime cognitive engagement and low beta-amyloid deposition. Arch Neurol, 69(5), 623-629.

Logan, J.M., Sanders, A.L., Snyder, A.Z., Morris, J.C., \& Buckner, R.L. (2002). Under-recruitment and nonselective recruitment: Dissociable neural mechanisms associated with aging. Neuron, $33(5), 827-840$

Mitchell, D.B. (2006). Nonconscious priming after 17 years invulnerable implicit memory? Psychol Sci, 17(11), 925-929.

Mueller, A.E., Raymond, N., \& Yochim, B.P. (2013). Cognitive activity engagement predicts future memory and executive functioning in older adults. Act Adapt Aging, 37(3), 251-264.

Nyberg, L., Lövdén, M., Riklund, K., Lindenberger, U., \& Bäckman, L. (2012). Memory aging and brain maintenance. Trends Cogn Sci, 16(5), 292-305.

Park, D.C., \& Bischof, G.N. (2013). The aging mind: Neuroplasticity in response to cognitive training. Dialogues Clin Neurosci, 15(1), 109-119.

Park, D.C., Lodi-Smith, J., Drew, L., Haber, S., Hebrank, A., Bischof, G.N., \& Aamodt, W. (2014). The impact of sustained engagement on cognitive function in older adults: The Synapse Project. Psychol Sci, 25(1), 103-112.

Park, D.C., \& Reuter-Lorenz, P. (2009). The adaptive brain: Aging and neurocognitive scaffolding. Annu Rev Psychol, 60, 173196.

Park, D.C., Smith, A.D., Morrell, R.W., Puglisi, J.T., \& Dudley, W.N. (1990). Effects of contextual integration on recall of pictures by older adults. J Gerontol, 45(2), P52-P57.

Reuter-Lorenz, P.A., \& Cappell, K.A. (2008). Neurocognitive aging and the compensation hypothesis. Curr Dir Psychol Sci, 17(3), 177-182.

Reuter-Lorenz, P.A., \& Park, D.C. (2014). How does it STAC up? Revisiting the scaffolding theory of aging and cognition. Neuropsychol Rev, 24(3), 355-370.

Robbins, T.W., James, M., Owen, A.M., Sahakian, B.J., McInnes, L., \& Rabbitt, P. (1994). Cambridge Neuropsychological Test Automated Battery (CANTAB): A factor analytic study of a large sample of normal elderly volunteers. Dementia, 5(5), 266-281. 
Sabsevitz, D.S., Medler, D.A., Seidenberg, M., \& Binder, J.R. (2005). Modulation of the semantic system by word imageability. Neuroimage, 27(1), 188-200.

Salthouse, T.A. (2006). Mental exercise and mental aging evaluating the validity of the "use it or lose it" hypothesis. Perspect Psychol Sci, 1(1), 68-87.

Schneider-Garces, N.J., Gordon, B.A., Brumback-Peltz, C.R., Shin, E., Lee, Y., Sutton, B.P., Maclin, E.L., Gratton, G., \& Fabiani, M. (2010). Span, CRUNCH, and beyond: Working memory capacity and the aging brain. J Cogn Neurosci, 22(4), 655-669.

Shors, T.J. (2014). The adult brain makes new neurons, and effortful learning keeps them alive. Curr Dir Psychol Sci, 23(5), 311318 .

Shors, T.J., Anderson, M.L., Curlik, D.M., \& Nokia, M.S. (2012). Use it or lose it: How neurogenesis keeps the brain fit for learning. Behav Brain Res, 227(2), 450-458.

Singh-Manoux, A., Richards, M., \& Marmot, M. (2003). Leisure activities and cognitive function in middle age: Evidence from the Whitehall II study. J Epidemiol Community Health, 57(11), 907-913.

Stine-Morrow, E.A.L., \& Basak, C. (2011). Chapter 10-Cognitive Interventions. In: K.W. Schaie \& S.L. Willis (Eds.), Handbook of the Psychology of Aging (Seventh Edition) (pp. 153-171). San Diego: Academic Press.

Wilson, R.S., Barnes, L.L., \& Bennett, D.A. (2003). Assessment of lifetime participation in cognitively stimulating activities. J Clin Exp Neuropsychol, 25(5), 634-642.

Wilson, R.S., Mendes De Leon, C.F., Barnes, L.L., Schneider, J.A., Bienias, J.L., Evans, D.A., \& Bennett, D.A. (2002). Participation in cognitively stimulating activities and risk of incident Alzheimer disease. JAMA, 287(6), 742-748.

Wilson, R.S., Scherr, P.A., Schneider, J.A., Tang, Y., \& Bennett, D.A. (2007). Relation of cognitive activity to risk of developing Alzheimer disease. Neurology, 69(20), 1911-1920.

Wirth, M., Haase, C.M., Villeneuve, S., Vogel, J., \& Jagust, W.J. (2014). Neuroprotective pathways: Lifestyle activity, brain pathology, and cognition in cognitively normal older adults. Neurobiol Aging, 35(8), 1873-1882.

Wirth, M., Villeneuve, S., La Joie, R., Marks, S.M., \& Jagust, W.J. (2014). Gene-environment interactions: Lifetime cognitive activity, APOE genotype, and beta-amyloid burden. J Neurosci, 34(25), 8612-8617. 